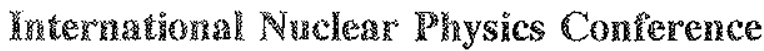

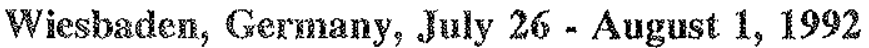

(To be published in Nucl.Phys. A)

\title{
Nuclear structure at the drip lines
}

\author{
P.G. Hansen \\ Institute of Physics and Astronomy, University of Aarhus \\ DK-8000 Aarhus C
}

\begin{abstract}
The drip-line nuclei under certain circumstances form a neutron or proton halo with a mass distribution that extends far outside the nuclear core. Examples are given of how this structure manifests itself through nuclear reaction cross-sections and angular distributions. A comparison with related phenomena in atoms and molecules is given.
\end{abstract}

\section{THE LIMITS OF NUCLEAR STABMLTTY}

The isobaric lines across the nuclear chart are terminated by instability towards the emission of nucleons. The two limits, to the north-west and south-east, correspond roughly to zero binding for protons and neutrons and are commonly. referred to as the drip lines. During the last years there has been a rapid development in our ability to investigate nuclei close to the drip lines thanks to a variety of techniques involving radioactive beams from isotope separators and recoil spectrometers. The proceedings [1] of a recent conference and also the review [2] give a good overview of activities in this field, which is right now subject to an intense interest. Many new results were presented at the 1992 Bernkastel-Kues Conference [3]. In this presentation I shall on the neutron drip line where novel phenomena have appeared, but even to this sub-sub specialty I cannot do full justice, especially with respect to the very many interesting theoretical papers.

\subsection{The proton drip line}

Owing to the Coulomb repulsion it is not possible to have nuclei with a very large proton excess and for the lighter elements the proton drip line lies rather close to the $N=Z$ line. The Coulomb barrier localizes a loosely bound or even unbound proton in the nuclear core and tends to make its wave function resemble that of a normally bound state. The combination of Coulomb- and angularmomentum barriers can lead to long lifetimes for proton emission from unbound states so that the proton drip line is overstepped. This is the phenomenon of proton radioactivity, which has been observed in a number of cases from iodine and up to rhenium [4]. Nearer to the stability line the energy spectra of protons emitted following beta decay give rich possibilities for studying the level structure up to 
relatively high energies and cases of beta-delayed two-proton emission have been reported, see e.g. [5]. It is striking that the proton-emitting resonances remain narrow up to quite high excitation energies, as shown for example by the decays [6] of the drip-line nuclei ${ }^{32,33} \mathrm{Ar}$ and even the beta decay [7] of ${ }^{17} \mathrm{Ne}$ leading to ${ }^{16} \mathrm{O}+\mathrm{p}$. The general experience is that the shell model describes the proton-rich nuclei rather well so that this region is "nuclear physics as usual".

\subsection{The neutron drip line}

The main part of this presentation is concerned with the neutron drip line. Since neutron matter in itself is close to being bound it is not surprising that a very large neutron excess is possible; according to some extrapolations of nuclear mass formulas ${ }^{176} \mathrm{Sn}$ is likely to be bound! Experimentally it is at present possible to reach the neutron drip line only for the lightest elements, where very striking effects appear. The essential new ingredient is that the low binding of the last neutron (or pair of neutrons), will permit quantum-mechanical tunneling of the particle(s) away from the nuclear core and hence the formation of a cloud or "halo" [8]. The result of this is that some almost sacred principles of nuclear physics are violated: (i) Mass and charge radii may differ by large amounts. (ii) Since the neutrons and protons are physically separated, electric dipole excitations of essentially single-particle strength appear at very low energy in dramatic contrast to the usual dominance of the Giant Dipole Resonance. (iii) Similarly, in beta decay there also seems to occur a re-emergence of "super-allowed" transitions, i.e. transitions with essentially single-particle strength. Finally, (iv) there are theoretical indications that three-body aspects play a role, leading to molecular or cluster features that are not fully accounted for in a mean-field approximation.

The following is a survey of the phenomenology of the neutron halo. I begin by presenting evidence that a halo nucleus can be viewed to a good approximation as consisting of two separate and almost independent systems, the core and the halo and I give examples of experimental techniques that probe one aspect or the other. This is followed by a brief digression to atomic and molecular physics to make a comparison with other loosely bound systems in quantum physics. After this I discuss in more detail studies of the halo via nuclear reactions leading to the continuum.

\section{THE NEUTRON HALO AS AN INDEPENDENT SUB-SYSTEM}

The neutron-rich nuclei that terminate the isobaric chains with masses $6,8,11$, 14 have an even number of neutrons and show to a larger or smaller degree evidence for a two-neutron halo. The most striking case is that of ${ }^{11} \mathrm{Li}$ which has a two-neutron separation energy $\mathrm{S}_{2 \mathrm{n}}$ of only $315 \pm 40 \mathrm{keV}$ as an average of three measurements [9]. There is only one known case of a single-neutron halo, that of ${ }^{11} \mathrm{Be}$, for which the odd neutron is bound by $502 \mathrm{keV}$.

There is mounting evidence that a halo nucleus is well approximated by a product wave function. We begin with experiments that demonstrate that the core is little affected by the presence of the halo neutrons. The magnetic dipole moments 
[10] of ${ }^{9} \mathrm{Li}$ and ${ }^{11} \mathrm{Li}$ have been measured by a refined combination of optical and beta-decay techniques to be 3.439 and 3.668 nuclear magnetons, respectively, close to the Schmidt limit of $3.71 \mathrm{n} . \mathrm{m}$. , and indicating that the proton system is little affected by the two additional neutrons in ${ }^{11} \mathrm{Li}$. Recently, it has been shown [11] that also the electrical quadrupole moments also are identical within the experimental errors: $-27.4 \pm 1.0$ and $-31 \pm 5 \mathrm{mb}$, respectively. Blank et al. [12] measured charge-changing reactions for ${ }^{8,9,11} \mathrm{Li}$ impinging on a carbon target and found that all have the same cross section.

The same experiment found a strong increase in the total cross-section (Fig. 1) similar to the one found at $800 \mathrm{MeV} / \mathrm{u}$ [13]. The strong energy dependence of the nucleon-nucleon cross-sections has been exploited in an experiment by Fukuda et al. [14], who demonstrated that the total reaction cross-sections for reactions of ${ }^{11} \mathrm{Be}$ at energies of 33 and $790 \mathrm{MeV} / \mathrm{u}$ with targets of $\mathrm{C}$ and $\mathrm{Al}$ could be understood only if it was assumed that the ${ }^{11} \mathrm{Be}$ density distribution had a long tail, the neutron halo. The calculated density distribution of a halo nucleus is shown in Fig. 1 taken from ${ }^{11} \mathrm{Li}$ calculation by Sagawa [15]. The momentum distributions of fragments also suggest the existence of the neutron halo. Kobayashi et al. [16] found that the transverse momentum distribution of ${ }^{9} \mathrm{Li}$ recoils from fragmentation of ${ }^{11} \mathrm{Li}$ on a carbon target is narrow and could be approximated as a superposition of two components with Gaussian shape ${ }^{a}$. Similar results were obtained for ${ }^{11} \mathrm{Be}$. The narrow momentum component is qualitatively in support of the picture of a halo: From Heisenberg's uncertainty principle it follows that a broad spatial distribution must be linked to a narrow momentum distribution.

There are also observables that are mainly sensitive to the halo. It was predicted [8] that Coulomb stripping plays an important role for high-Z targets and at low energies since the cross-section varies at intermediate energies approximately as $\mathrm{Z}^{2} /\left(\mathrm{S}_{11} \mathrm{E}_{0}\right)$, where $\mathrm{E}_{0}$ is the beam energy per nucleon. The process was first observed by Kobayashi et al. [18] at $800 \mathrm{MeV} / \mathrm{u}$ in experiments where ${ }^{8} \mathrm{He},{ }^{11} \mathrm{Li}$ and ${ }^{14} \mathrm{Be}$ collided with a lead target. At this high energy only $15-30 \%$ of the total crosssection is electrical. However, at $29 \mathrm{MeV} / \mathrm{u}$ [19] it becomes dominant (see also Section 4), so that for a gold target the experimental cross section for the removal of two neutrons from ${ }^{11} \mathrm{Li}$ is $5.0 \pm 0.8 \mathrm{~b}$, two orders of magnitude above "normal" Coulomb excitation cross-sections. This is evidence for the appearance of the E1

" As there has been some debate about the parametrization of momentum distributions, a few comments on this may be useful. It is occasionally assumed that the two Gaussians are evidence for two separate mechanisms and in ref. [1] on p. 233 there is experimental "proof" that a single Lorentzian will not fit the experimental data. (It will, in fact, quite nicely. Try something like $\left.1000 /\left(1+\left(\mathrm{p}_{\lrcorner} / 46\right)^{2}\right) \ldots\right)$ The point that should be kept in mind is that any simple theoretical concept has a certain domain of validity. The halo, introduced to explain effects at large distances and, consequently, at small momenta, will not necessarily account for what happens at momenta of $100-200 \mathrm{MeV} / \mathrm{c}$, corresponding to nuclear dimensions, let alone at higher momenta. The choice of any simple function, therefore, is essentially a matter of convenience in the presentation. 


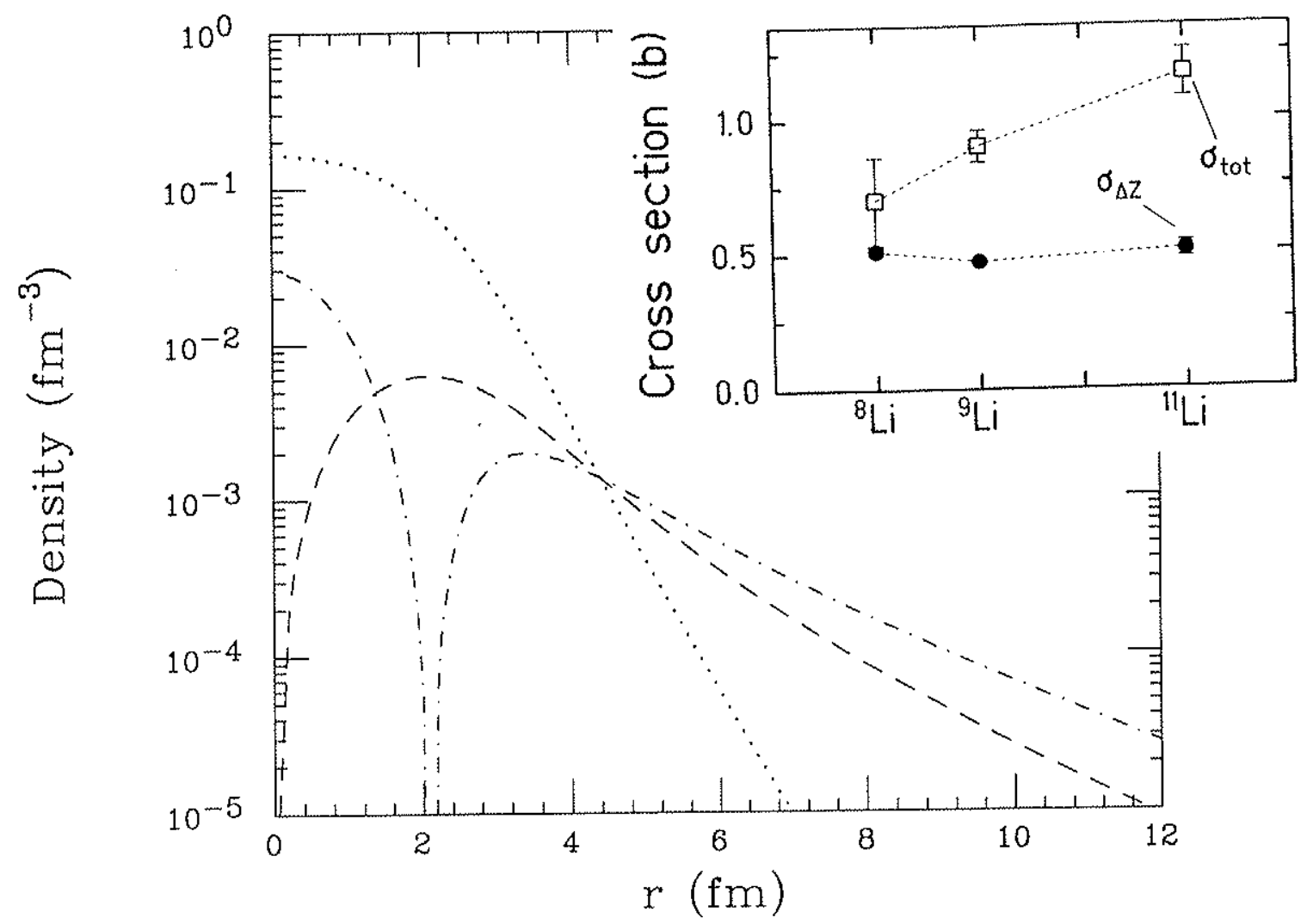

Figure 1. Inset: Charge-changing and total cross-sections [12] for $80 \mathrm{MeV} / \mathrm{u}^{8,9,11} \mathrm{Li}$ incident on a carbon target. Main figure: Density distributions in ${ }^{11} \mathrm{Li}$ for the core and the halo neutrons (assumed to be in either $1 \mathrm{~s}_{1 / 2}$ or $0 \mathrm{p}_{1 / 2}$ states) according to a Hartree-Fock calculation by Sagawa [15]. Note that the halo constitutes a very dilute neutron gas, a neutron "stratosphere".

transition probabilities of the order of a single particle unit referred to in Section 1. Not only Coulomb stripping but also nuclear stripping is strongly enhanced for a nucleus with a neutron halo. Thus for $29 \mathrm{MeV} / \mathrm{u}$ ions incident on a beryllium target the neutron yield near zero degrees [19] is more than an order of magnitude greater for ${ }^{11} \mathrm{Li}$ with 8 neutrons than it is for ${ }^{9} \mathrm{Li}$ with six neutrons. Likewise it is found at $33 \mathrm{MeV} / \mathrm{u}$ [14] that the fragmentation cross section ${ }^{11} \mathrm{Be}+\mathrm{Al} \rightarrow{ }^{10} \mathrm{Be}+\mathrm{X}$ of $0.65 \pm 0.05 \mathrm{~b}$ exceeds that of the corresponding stripping reaction of ${ }^{18} \mathrm{C}$ by a factor of 7 .

Tanihata et al. [20] have pointed to an empirical additivity law for the interaction cross-sections of the helium isotopes. It is seen from Table 1 that the increase in the interaction cross-sections at $800 \mathrm{MeV} / \mathrm{u}$ with increasing mass number is explained essentially by the neutron removal cross-sections. The oneneutron removal cross-section for ${ }^{11} \mathrm{Be}$ is noted to obey the same law, which has been justified theoretically by Yabana et al. [21], who performed calculations of ${ }^{11} \mathrm{Li}$ 2 n-dissociation cross-sections on a target of ${ }^{12} \mathrm{C}$ in a four-body (core, target, two neutrons) model and on the basis of an optical model for the nucleus-neutron interaction. Their results are in good agreement with the measured value [19] at 
$29 \mathrm{MeV} / \mathrm{u}$ and for a beryllium target. It is interesting that the elastic and nonelastic contributions are nearly identical at the lower energies $\left(\mathrm{E}_{0}<100 \mathrm{MeV} / \mathrm{u}\right)$ exactly as expected from a simple black-disc model.

Table 1

Interaction $\left(\sigma_{\mathrm{l}}\right)$ and neutron-removal $\left(\sigma_{-\mathrm{xn}}\right)$ cross-sections in $\mathrm{mb}$ and at $800 \mathrm{MeV} / \mathrm{u}$

\begin{tabular}{llll} 
Reaction & $\sigma_{1}$ & $\sigma_{-2 n}$ & $\sigma_{-4 n}$ \\
\hline${ }^{4} \mathrm{He}+{ }^{12} \mathrm{C}$ & $503 \pm 5$ & & \\
${ }^{6} \mathrm{He}+{ }^{12} \mathrm{C}$ & $722 \pm 5$ & $189 \pm 14$ & \\
${ }^{8} \mathrm{He}+{ }^{12} \mathrm{C}$ & $817 \pm 6$ & $202 \pm 17$ & $95 \pm 9$ \\
\hline
\end{tabular}

Finally it has been noted [22] that even-n nuclei at the neutron drip line show fast ("superallowed") beta transitions with a strength and energy that may be interpreted as arising from the decay of a quasi-free pair of neutrons. It is also tempting to view the very strong triton branch of $0.8 \%$ [23] in the decay of ${ }^{8} \mathrm{He}$ as a fragment from the decay of a tetraneutron. However, the beta decay evidence for the halo is on a much less firm footing than that from reactions.

In Section 4 we shall return to nuclear reactions of halo nuclei and consider in more detail the interplay between nuclear structure and reaction mechanism. Since the halo brings some qualitatively new features into nuclear physics, it seems interesting briefly to consider whether related phenomena exist at the atomic and molecular level.

\section{MARGINALLY BOUND ATOMIC AND MOLECULAR SYSTEMS}

The formation of the neutron halo is caused by an interaction that is weak and of short range. Furthermore, the di-neutron halo owes its stability as much to the neutron-neutron interaction as to the core-neutron interaction. We look for systems at the atomic level that possess some of these features, and note first that "atoms" do not change structure as a function of their binding strength. Even if we, as a thought experiment, allow the strength $e^{2}$ of the electromagnetic interaction to become a continuous variable, no qualitatively new features will appear. This can be seen immediately from the non-relativistic Hamiltonian for an $n$-electron system coupled to an infinitely heavy core with charge $Z$, which leads to an eigenvalue equation with no free parameters if length is measured in units of the Bohr radius $a=\hbar^{2} / m e^{2}$ and energy in units of $m e^{4} / \hbar^{2}$. All "atoms" of a given stoichiometry thus have the same structure.

The two-electron atom presents an interesting analogy to the two-neutron halo. It has been known since Hylleraas explained the helium atom in 1929 (for an account of this and later developments see e.g. [24]) that a product of two identical wave functions underestimates the binding by some $1.4 \%$. This deviation may be accounted for by introducing coordinates that contain the distance between the two 
electrons ("correlations") so that probability of finding the two electrons close to each other is smaller than in an independent-particle scheme. The stability of the simplest two-electron atom, the negative hydrogen ion, is entirely caused by the electron-electron correlations. We must expect that similar effects (although with opposite sign since the $n n$ force is attractive) will be important for the two-neutron halo nuclei.

The next Sections will mainly deal with the question how to obtain information about the structure of a bound state (the halo) from inelastic scattering reactions leading to continuum states. A particularly transparent illustration of this comes from an atomic physics experiment by Lohmann and Weingold [25], who used the $(e, 2 e)$ reaction on hydrogen atoms to re-construct the momentum distribution of the struck electron. At high bombarding energies, under their conditions corresponding to beam energies of $400-1200 \mathrm{eV}$, the result is independent of the bombarding energy and gives the (already known) true result for the momentum distribution.

An "atom" consisting of three unit charges with different masses and two like charges has a more complex behaviour. This problem has recently been discussed by Martin et al. [26], who give references to a number of experimental and theoretical papers on the subject. It turns out that symmetric atoms always are stable, such as the hydrogen molecule ion $\mathrm{H}_{2}{ }^{+}$, the negative hydrogen ion $\mathrm{H}^{-}$, and the more recently discovered [27] trielectron $e^{+} e^{-} e^{-}$. Taking particles 2 and 3 to be those that have opposite sign from that of particle 1, Martin et al. show that it is useful to introduce variables $\alpha_{i}$ proportional to the reciprocal masses of the particles and with their sum normalized to unity. Hence, the stability problem is determined by the choice of two values of the $\alpha_{i}$ so that the region of stable systems can be represented in the triangle diagram shown in Fig. 2. The borders of the hatched area are the "drip lines" of such systems.

Consider now potentials that decrease faster with distance than $1 / r$. A particle bound in a potential that varies with distance as $r^{-2}$ corresponds to the limiting case [28] in which the particle is either unbound or falls to the origin, depending on the strength of the interaction. Such a potential is realized approximately for an electron bound in the field of a (finite) dipole, and it has been shown [29] that the limit to binding is represented by the dipole moment 0.639 in atomic units. Bound states of this kind have been observed by Lykke et al. [30] as photodetachment resonances of the acetaldehyde enolate negative ion $\left(\mathrm{H}_{2} \mathrm{C}-\mathrm{COH}{ }^{-}\right)$. The ground state of this band is bound against autoionization by only $0.5 \mathrm{meV}$ and already the second rotational state decays by spontaneous emission of electrons. These states can be estimated to be quite large, of the order of $100 \AA$ and represent an atomic halo state.

Systems held together by attractive forces of even shorter range, are exemplified in molecular physics by the van der Waals force between noble gas molecules. It is a common feature of the three-body problem with short-range forces $[31,32]$ that it is possible to have a bound state, even if none of the three two-body sub-systems are bound. One possible candidate for this behaviour is the He atom, for which the molecule $\mathrm{He}_{2}$ is believed to be unbound, while $\mathrm{He}_{3}$ could possibly be stable at low temperatures. The general interest in the three-body problem with weak shortrange attrective forces has been stressed by Efimov [31], who points out that 
Figure 2

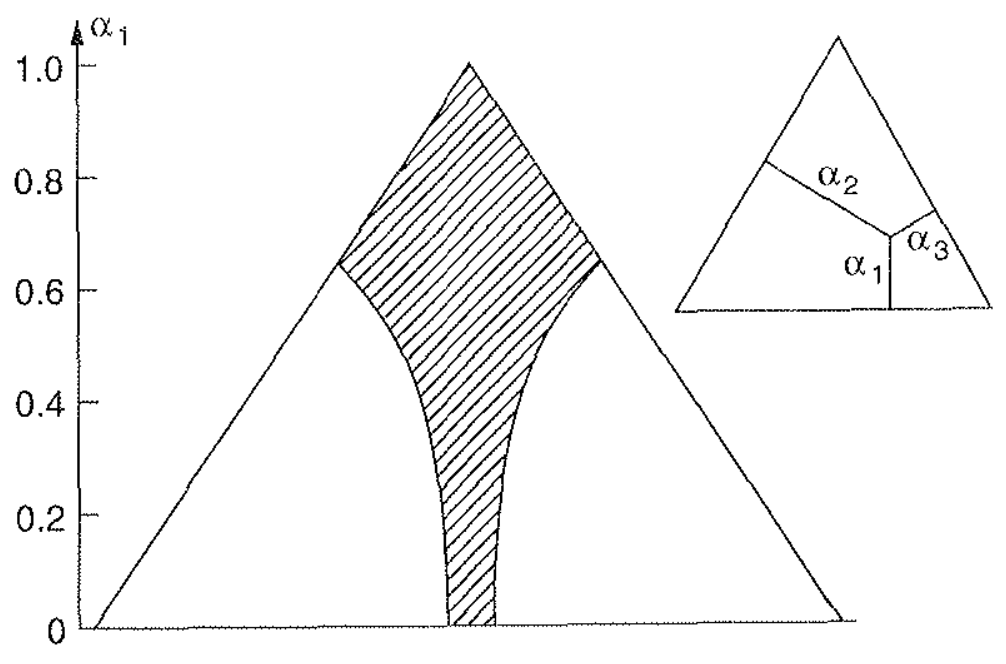

The hatched area shows the estimate by Martin et al. of the region of stability for a three-particle Coulomb system as a function of the normalized inverse masses represented in the triangle diagram as shown in the inset.

although it is usually believed that the three-body problem is too complex to be analyzed in qualitative terms, there exists, in fact, for weakly bound systems a rather wide class of tractable solutions. These fulfill two conditions, namely that the size of the two-body system, represented by the magnitude of the scattering length $a$ should be much larger than the range $R$ of the force (this is the condition for having a resonance near zero energy), and secondly that the energy of the system should be much smaller than the Wigner limit $|\mathrm{E}|<\hbar^{2} / m R^{2}$. Efimov has shown that in the limit of very large systems, a series of very loosely bound threebody levels arises. The best chance for probing such systems must lie in nuclear physics, which offers many examples of bound three-body systems for which neither of the three two-body sub-systems are bound.

From this point of view, ${ }^{11} \mathrm{Li}$ seems to offer an almost ideal candidate since both sub-systems are close to resonant. It is well known that the $n n(S=0, T=1)$ resonance lies at $70 \mathrm{keV}$, only, and recent experiments [33] on (the unbound) ${ }^{10} \mathrm{Li}$ find resonances near zero energy.

\section{STRUCTURE AND REACTIONS ON THE ONE-NEUTRON HALO}

In this and the following Section we examine how the strikingly narrow transverse momentum distributions discussed in Section 2 can contribute to the understanding of the neutron halo. This approach is one that is forced upon us by necessity. The time-honoured road to insight into nuclear structure at low energies is that of measuring the energies and angular-momentum assignments of as many low-lying levels as possible and to deduce structure and dynamics from this cryptogram. This method will not work for the halo nuclei, which most commonly have only one bound state, the ground state, in addition to a broad and largely featureless continuum. Thus, if we want to go beyond the gross features discussed 


\section{Figure 3}

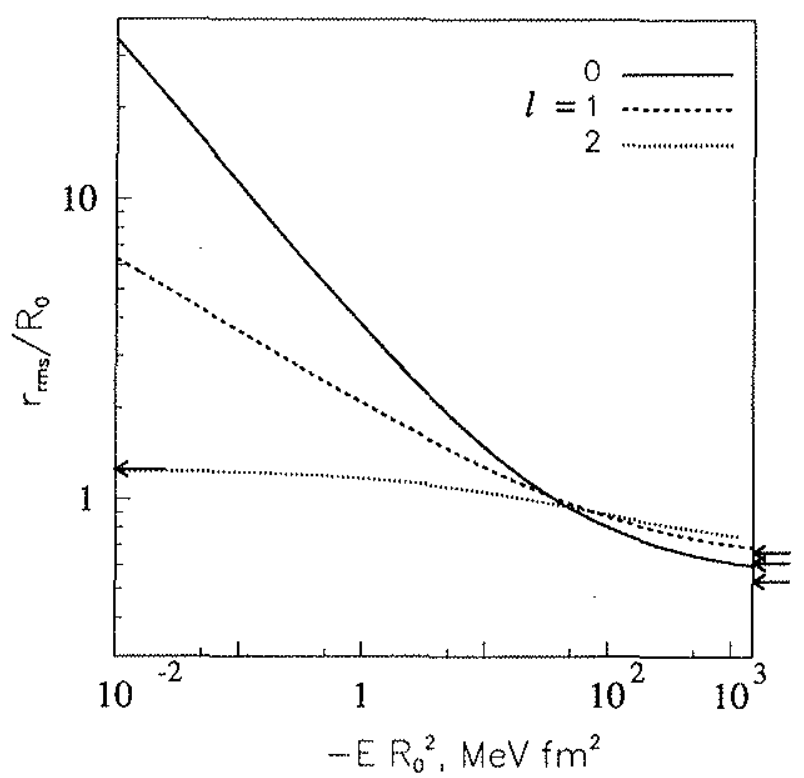

For a square-well potential with radius $R_{0}$ and depth $U_{0}$ the root-mean-square radius is shown as a function of the binding energy in appropriate units. The reduced mass of the system was taken to be 10/11 times the neutron mass. The arrows indicate the limiting values derived analytically for zero and infinite binding.

in Sect. 2, we must be willing to study processes that excite the continuum. We know that the halo is weakly coupled to the core and therefore have all reasons to expect that the continuum will feel this coupling even less, in short that it will resemble free particles mightily. As this Section will show, this is not such a bad starting point. For this reason I disagree mildly with the terminology of those, who in the low-lying E1 excitations of the halo see "soft, micro or mini" Giant Dipole Resonances and the like. I agree that we are all hoping to see features that go beyond what we already know from the photodisintegration of the deuteron, but we deceive ourselves if we do not admit that the continuum excited with essentially single particle strength is there as a very good starting point.

The difficulty encountered in probing the halo in more detail by means of nuclear reactions lies in the interplay between nuclear structure and the reaction mechanism. Before we turn to the two-neutron halo in the following Section, we therefore examine the case of ${ }^{11} \mathrm{Be}$ for which the structure part is well understood.

\subsection{The one-neutron halo and ${ }^{11} \mathrm{Be}$}

The interesting general question whether a short-range attractive and weak potential can develop a halo structure for any value of the angular momentum has recently been answered by Riisager et al. [34]. They investigated the behaviour of the moments $\left\langle r^{n}\right\rangle$ of the wave function as the neutron separation energy decreases towards zero and found the moment to be finite provided that $n<2 l-1$. Hence, if a halo is defined as a divergent second moment of $r$ as a function of $\mathrm{S}_{\mathrm{n}}$, then this occurs only for $s$ and $p$ states. This property appears clearly from numerical 
calculations carried out with a square-well potential, see Figure 3. Similar observations have been made by Sagawa [15], who remarks that states with $l>1$ "are not likely to form a halo". Riisager er al. also investigated the properties of a two-body proton halo as a function of the proton binding; as could be expected the Coulomb barrier reduces the halo considerably, but appreciable effects still remain for $s$ states of light muclei.for a neutron $s$ state the external wave function is simply a Yulkawa proportional to $\exp (-r / \rho) / r$ where the decay length

$$
\rho=\frac{\hbar}{\sqrt{2 \mu S_{n}}}
$$

is given in terms of the reduced mass and the neutron separation energy.

The ground state of ${ }^{11} \mathrm{Be}$ was early recognized [35] as an example of what we today call an intruder state, a $1 s_{1 / 2}$ state that for $\mathrm{N}=7$ and small proton numbers dives below the $0 p_{1 / 2}$ state. Modern shell-model theory, see Otsuka et al. [36] views the state as predominantly a combination of this s-state with a $d_{5 / 2}$ coupled to the soft $2^{+}$of ${ }^{10} \mathrm{Be}$, thus making the link to the Nilsson model. The neutron separation energy given in Section 2 leads to the external decay length $\rho=6.8 \mathrm{fm}$, well in excess of the root-mean-square radius of a $p$-shell nucleus of about $2.5 \mathrm{fm}$. The $0 p_{1 / 2}$ state lies at $320 \mathrm{keV}$ and its lifetime was measured by Millener et al. [37], who
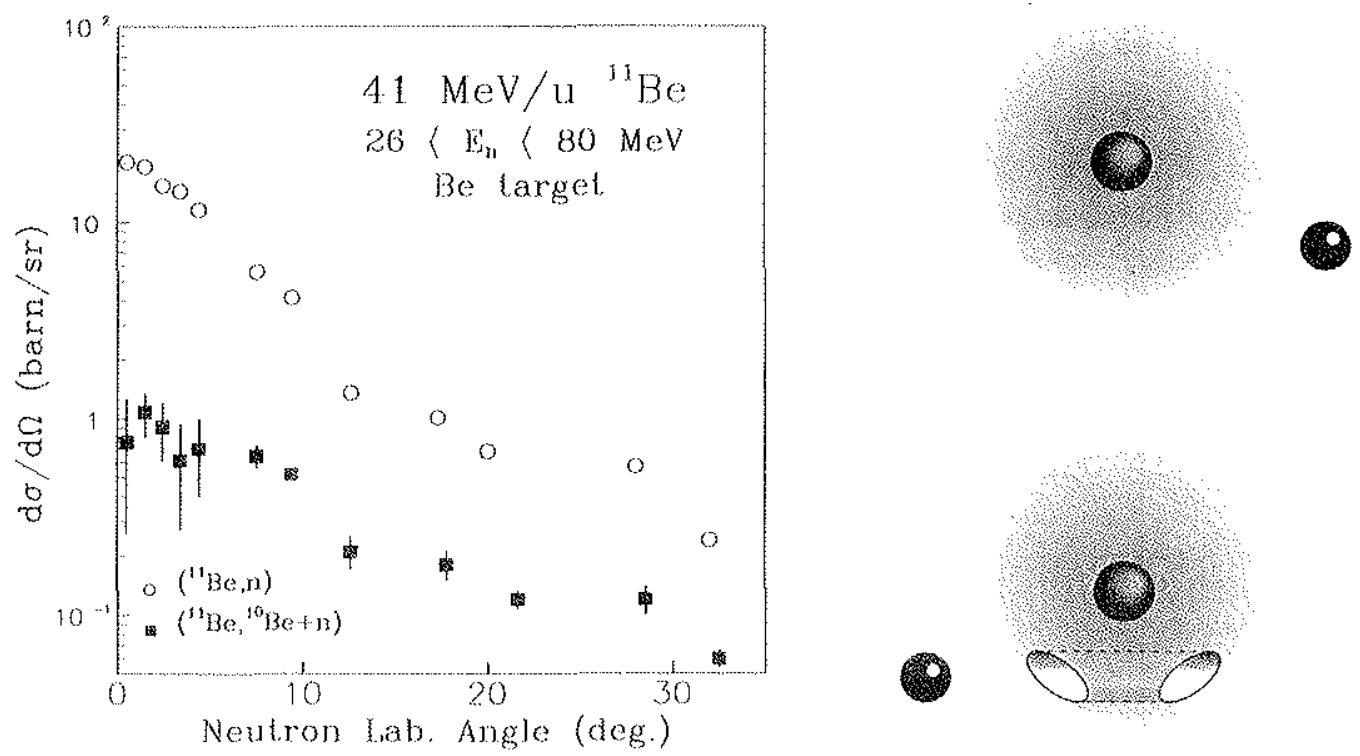

Figure 4

The single neutron inclusive and exclusive $\left(n+{ }^{10} \mathrm{Be}\right)$ reaction cross-sections [54] for $41 \mathrm{MeV} / \mathrm{u}{ }^{11} \mathrm{Be}$ incident on a beryllium target. The figure to the right illustrates the Glauber model [55] of absorption and diffraction dissociation. Seen from the projectile (halo) coordinate system, the incident target nucleus traverses the halo (from the right) and causes either absorption or a "wound" in the wave function. The latter implies a certain probability of disintegration. For high-energy reactions, i.e. beam velocity much higher than the halo velocity, cross-sections and momentum distribution can be calculated from this simple geometrical model. 
found that it is the fastest $\mathrm{E} 1$ between low-lying states known in nuclear physics: the reduced transition probability is 0.36 Weisskopf units. The link between the neutron binding energy, nuclear size and transition probability was realized in this work, which represents the first identification of a neutron halo.

\subsection{Coulomb and nuclear dissociation of ${ }^{11} \mathrm{Be}$}

From the experiments discussed in Section 2 we know that the dissociation reactions that leave the nuclear core untouched give a very clear halo signal. The essential elements in these reactions, Coulomb dissociation for high- $\mathrm{Z}$ targets and absorption and diffraction dissociation for light targets, have been discussed in many theoretical papers, some mainly oriented towards the nuclear process [38-43] and some mainly towards the Coulomb process [43-53]. A new experiment [54] studied the one-neutron dissociation reaction by detecting a fast forward neutron

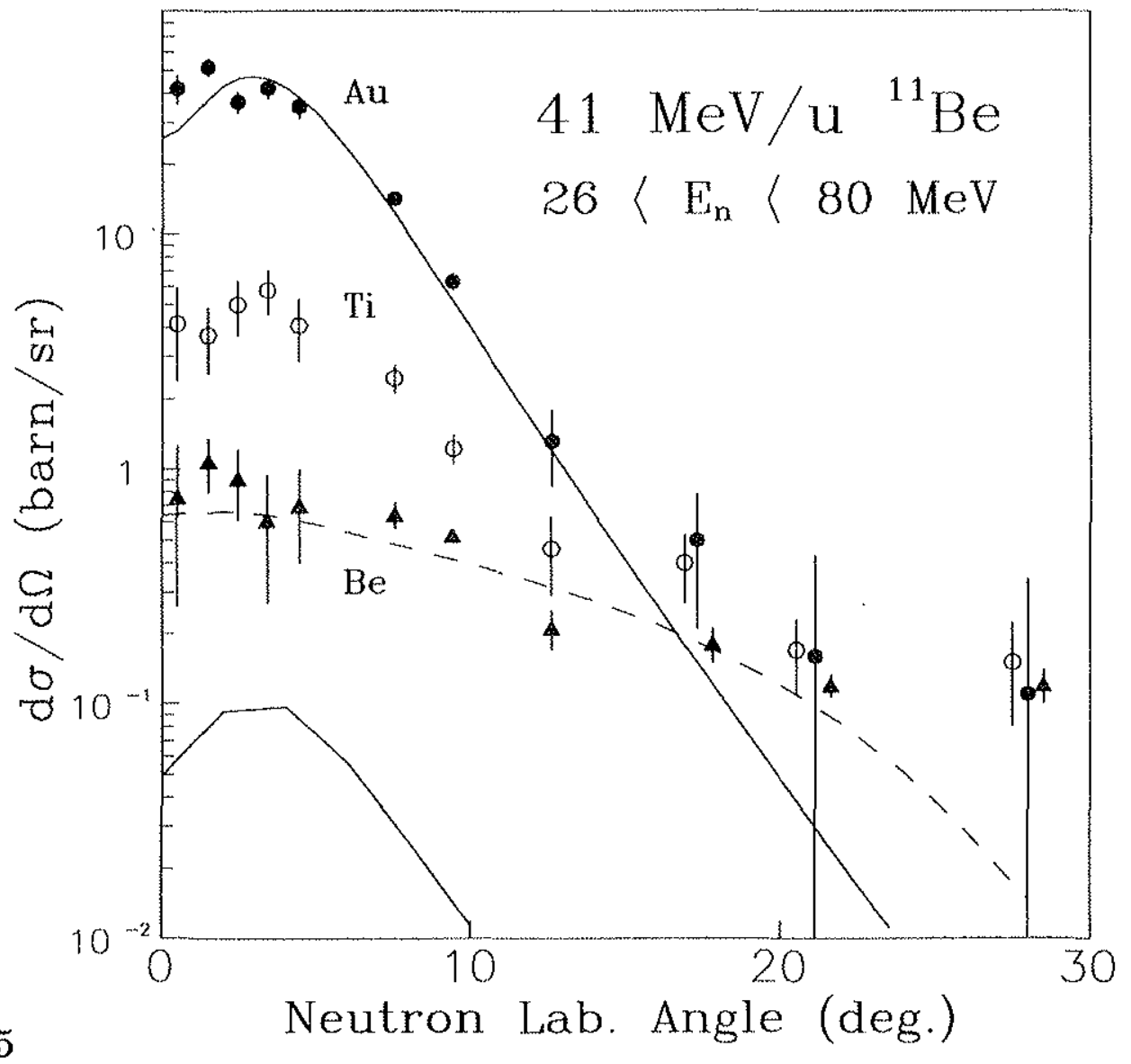

Figure 5

The exclusive cross-sections for ${ }^{11} \mathrm{Be}$ at $41 \mathrm{MeV}$ (average of entrance and exit energy in the target) incident on targets of $\mathrm{Be}, \mathrm{Ti}$ and $\mathrm{Au}$ and compared with a schematic ealculation, which serves to identify the essential ingredients in the reactions. The calculated differential cross-sections for Coulomb excitation of $\mathrm{Au}$ and Be are shown as full drawn lines and the calculated angular distribution arising from diffraction dissociation on $\mathrm{Be}$ is shown as a dashed line. There are no free parar eters in these calculations, which use the same simple wave function. 
in coincidence with a ${ }^{10} \mathrm{Be}$ recoll. For a a heavy target such as gold these exclusive differential cross-sections are not very different from the single neutron inclusive distributions, whereas for the beryllium target (Figure 4) there is a vast diffexence and the exclusive distribution is very broad as is expected for diffraction dissociation on a small target nucleus. The very much larger inclusive cross-section reflects contributions from core-core collisions: (i) a broad contribution of fass neutrons from core fragmentation and punch-through of the light target, and (ii) a narrow distribution coming from the halo neutron.

The exclusive angular distributions are shown in Figure 5 together with the results of two simple calculations, both based on a Yukawa wave function alone in the "zero range approximation", and in which the cross-sections resulting from an integration over impact parameter were augmented by the finite-size correction factor [8]. The Coulomb calculation followed in principle closely Baur and his collaborators [44,48,49] and nuclear contributions were neglected.

For the case of a beryllium target the Coulomb cross-section is much too small to explain the experimental result although it has been included with the calculated nuclear contribution (dashed), essentially a black-disc model in the spirit of the calculation of R.J. Glauber [55] of the absorption and diffraction dissociation of the deuteron. The broad distribution found for (exclusive) neutrons on $\mathrm{Be}$ corresponds very approximately to (see Section 5.1) $\Gamma=130 \mathrm{MeV} / \mathrm{c}$; qualitatively this is in agreement with Kobayashi et al. [56] who at $800 \mathrm{MeV} / \mathrm{u}$ found $180 \mathrm{MeV} / \mathrm{c}$, a result that was intepreted [19] in favour of the diffraction mechanism.

\section{THE TWO-NEUTRON HALO AND ${ }^{11} \mathrm{Li}$}

The structure of ${ }^{11} \mathrm{Li}$ has been investigated theoretically by a number of the methods available in the theorist's arsenal, shell model (see especially the review by Brown [57]), cluster model, three-body calculations, Hartree-Fock calculations etc. [58-67, 36] and also in connection with the many reaction studies cited elsewhere in this paper. A very essential step, later enshrined by many others, was taken by Bertsch, Brown and Sagawa [58], who adjusted the strength of the interaction used in their Hartree-Fock calculations so that the experimental separation energy was reproduced for the last occupied orbit. The subsequent sequence of papers by Bertsch, Esbensen and their collaborators $[47,52,53,59,63]$ probably represent the most developed picture, so far, of the structure of "Li and its Coulomb interactions. Later in this section these calculations will be compared with the experimental results.

\subsection{Angular distribution of framents from the dissociation of "1:}

Although measurements for other, more bound, halo systems such as ${ }^{6,8}$ He and ${ }^{14}$ Be exist and demonstrate the qualitative link between binding of the halo and width of the momentum distributions, we concentrate here on the results for "Li. We begin by some remarks to define common ground. The Goldhaber model, which underlies much of the current thinking about high-energy fragmentation reactions, parametrizes the internal momentum distribution of a nucleus in terms of a 
parameter $\sigma$, the root-mean square momentum along one axis. The coresponding three-dimensional distribution is not changed by integration over two axes to obtain the transverse momentum distribution, given in some papers, so the the full width at half maximum is given by $\Gamma=2.35 \sigma$. Consider now a zero-range Yukawa wave function such as defined in Section 4.1, with a momentum distribution in three dimensions

$$
w(p)=\frac{\rho^{3} \hbar^{-3}}{\pi^{2}\left[1+(p \rho / \hbar)^{2}\right]^{2}}
$$

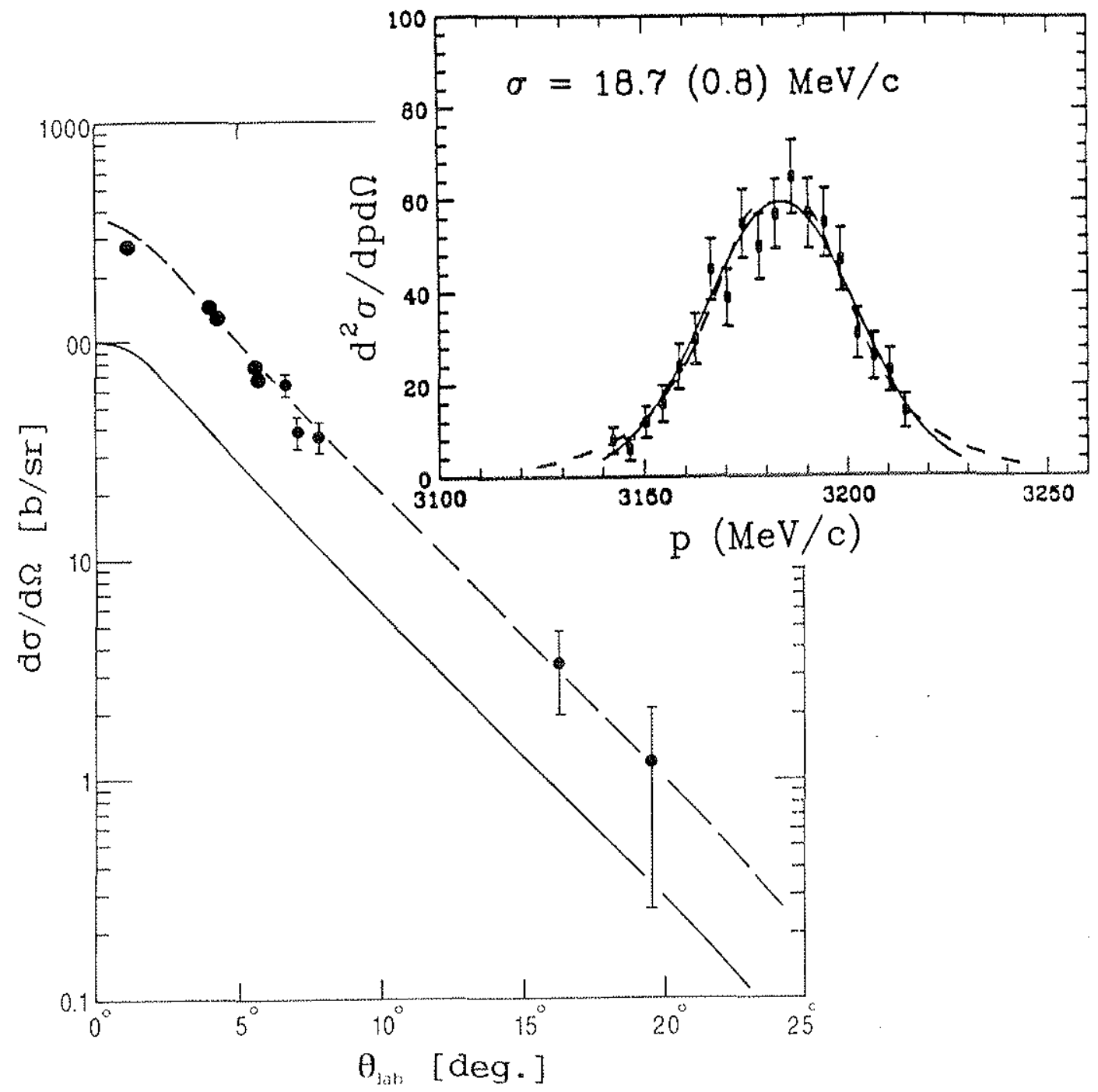

Figure 6

Experimental angular distribution of neutrons from the reaction ${ }^{11} \mathrm{Li}+{ }^{179} \mathrm{Au} \rightarrow$ ${ }^{9} \mathrm{Li}+\mathrm{n}+\mathrm{X}$ [19] at $29 \mathrm{MeV} / \mathrm{u}$ together with the calculated [52] values from Coulomb excitation, absolute (full drawn) and renormalized (dashed) values. The inset shows the longitudinal momentum distribution of ${ }^{9} \mathrm{Li}$ (arbitrary scale) from the $\mathrm{Ta}\left({ }^{11} \mathrm{Li}\right.$, $\left.{ }^{9} \mathrm{Li}\right)$ reaction [71], a Gaussian fit (full drawn) and the calculations by Esbensen and Bertsch [53] (dashed). 
which is simply the square of its Fourier transform. After integration over two axes this distribution corresponds to a Breit-Wigner shape with $\Gamma=2 \hbar / \rho$ whereas the corresponding angular distribution $\mathrm{do} / \mathrm{d} \Omega$ has $\mathrm{FWHM}=0.766 \Gamma$. We discuss all results in terms of a single effective $\Gamma$ parameter.

The original result of $\Gamma=90 \mathrm{MeV} / \mathrm{c}$ reported by Kobayashi et al. [16] for 800 $\mathrm{MeV} / \mathrm{u}^{9} \mathrm{Li}$ recoils from breakup of ${ }^{11} \mathrm{Li}$ on a carbon target was originally considered. a small value, but as will be seen from the following the main question today is why it is not even smaller. It is therefore important that the experiment has been repeated with better statistics and with identical results for a carbon target and for a hydrogen target $[9,68,69]$. The latter finding demonstrates that we are not dealing with an effect of Coulomb scattering.

The angular distribution of neutrons from the exclusive channel $n+{ }^{9} \mathrm{Li}$ from bombardments of targets of $\mathrm{Be}, \mathrm{Ni}$ and $\mathrm{Au}$ (Figure 6) with $29 \mathrm{MeV} / \mathrm{u}^{11} \mathrm{Li}$ gave essentially identical distributions $[19,70]$, which after correction for the detector size and beam divergence correspond to $\Gamma=26 \mathrm{MeV} / \mathrm{c}$. This result was surprising in two respects. First, it would be expected that, as subsequently demonstrated in the ${ }^{11}$ Be experiment discussed in the previous Section, the outcome would be different for light and heavy targets, since the reaction mechanisms are different in the two cases. This, together with the extreme narrowness of the distributions, invited the tentative conclusion [19] that the identity of the three distributions reflected their common feature, the momentum distribution of the halo ground state. The high intensity near zero degrees could then easily hide less distinctive features. But the second problem is that even with this assumption, the momentum width appears to be too small relative to the core recoil momentum width cited above, a conclusion that is also reached from an analysis that subdivides the distributions into broad and narrow components [69].

The transverse momentum distribution of the core recoil could possibly be influenced from Coulomb and maybe even nuclear contributions at small impact parameters. An experiment on the longitudinal momentum distribution of the ${ }^{9} \mathrm{Li}$ fragment from breakup of ${ }^{11} \mathrm{Li}$ at $66 \mathrm{MeV} / \mathrm{u}$ on targets of $\mathrm{Be}, \mathrm{Nb}$ and $\mathrm{Ta}$, recently completed by Orr et al. [71], avoids this difficulty since the Coulomb contributions from the in-and outgoing trajectory to lowest order cancel ${ }^{a}$. The result, in Figure 6 , is that the momentum distributions are essentially identical, just as in the neutron experiment. For a gold target an extrapolated o parameter of $18 \mathrm{MeV} / \mathrm{c}$ corresponds to $\Gamma=42 \mathrm{MeV} / \mathrm{c}$. This is in excellent agreement with what should be expected for an uncorrelated neutron pair: $\Gamma(2 \mathrm{n})=26 \cdot \sqrt{2}=37 \mathrm{MeV} / \mathrm{c}$.

Both experiments are for the heavy targets in good agreement with calculations $[59,63,52]$ that develop a three-body model and calculate the dipole strength function. An important feature of the calculation, also emphasized in a subsequent paper [53], is that the strength of the dipole excitation is enhanced by about $50 \%$ by the $n n$ correlations; this is opposite to ordinary nuclear correlations, which

\footnotetext{
"A slight Coulomb "post-acceleration" would be expected because the fragment is lighter but has the same charge. The experiment shows indications of this. The effect has been discussed by Baur [49].
} 
decrease the dipole strength by shifting it to higher energies. The model also provides a handle for understanding why the momentum distribution of the dipole excitations resembles the momentum distribution of the ground state.

\subsection{Other experiments on ${ }^{11} \mathrm{Li}$}

Unlike what is the case in atomic physics (Sect. 3) we do not understand the absolute binding of nuclei well enough to trace the effects of correlation in this way. The best chances may seem to be in the distribution of transition strengths or, maybe better, the angular correlations of $n n$ events [53], for which an, at first sight surprising, anticorrelation is predicted. This implies, roughly speaking, that the neutrons from a pair will most probably be found on opposite sides of the reaction axis. Experiments on neutron-neutron coincidences are in progress in several places. The first result [70] was based on a few hundred coincidence events, which within experimental errors were consistent with an uncorrelated distribution. Shimoura et al. [72] report a correlation corresponding to a relative energy of 50 $\mathrm{kV}$ of the neutrons.

Two experiments $[73,74]$ have investigated the angular distribution in elastic scattering of ${ }^{11} \mathrm{Li}$ on targets of hydrogen and silicon at 60 and $29 \mathrm{MeV} / \mathrm{u}$, respectively. The most striking feature is a decrease in cross-section relative to that of more bound lithium isotopes, clearly associated with breakup of the projectile and most pronounced for the heavy target.

In another experiment Kobayashi et al. [9] studied the pion double-charge exchange reaction ${ }^{11} \mathrm{~B}\left(\pi^{\prime}, \pi^{+}\right)^{11} \mathrm{Li}$ and found strong excitations near $1.2 \mathrm{MeV}$ representing mainly the single-particle continuum. As compared with other similar transitions to non-analogue states the ground-state transition appears to be hindered by a factor of about 0.3 , see theoretical discussions by Gibbs and Hayes [75] and also the footnote in Section 6.

\subsection{Where do we stand?}

Another phenomenon that would allow the undisturbed momentum distribution to emerge would be a sudden and violent impact that would remove the core. We are not so far from this limit. For the Coulomb collision of a halo nucleus with a target the violence of the process is measured by the product $q \cdot \rho$, where $q$ is the momentum transferred to the CM system of the halo nucleus by the Coulomb force, and where $\rho$ is the halo range parameter used previously in this paper. For a grazing collision of, say, a Be or Li ion with a heavy target this product is at 29 $\mathrm{MeV} / \mathrm{u}$ of order unity or greater so that perturbation theory is no longer applicable. This may contribute towards explaining why the total Coulomb cross-sections for ${ }^{11} \mathrm{Li}$ do not scale with the square of the target atomic number $\mathrm{Z}$ but rather with the power 1.41 \pm 0.22 [18]. Another explanation for this has been proposed by Suotome et al. [51], who attribute it to $\mathrm{Z}$ dependence of the lower momentum cut-off.

Conservation of isospin and the halo structures has been considered by Suzuki and Yaban: 176$]$, who conclude that isospin is conserved and that multiplets exist, also for the halo states.

All in all, we begin to understand the neutron halo and its reactions. Among the unanswere $\mathrm{d}$ questions are the transverse momentum distributions obtained for 
light targets: Why are they so broad for the core recoils and so narrow for the neutrons from ${ }^{11} \mathrm{Li}$ ?

\section{WHAT'S NEXT}

All the light nuclei at the neutron drip line show some measure of a halo structure. Most of the work until now has concentrated on the best cases, "Li and ${ }^{11} \mathrm{Be}$, but there must exist very interesting candidates beyond beryllium such as ${ }^{19} \mathrm{~B}$, ${ }^{19} \mathrm{C}$ (a candidate for one-neutron halo similar to ${ }^{11} \mathrm{Be}$ ), ${ }^{22} \mathrm{C}$ and so on. They have been produced in the laboratory but the experiments are hard and it will become even harder to match the precision obtained in the present generation of experiments, so intensity is the name of the game. It would be valuable if it were possible to perform simple experiments that directly would give a hint of the amount of halo in a given candidate. The key to this is, of course, the separation energy of the halo as the halo size scales with $\sqrt{ } S$. One possibility would be to detect with high efficiency the forward neutrons produced by the fragment on a heavy target: The calculations used in Fig. 5 indicate that the Coulomb peak crosssection scales as $1 / S^{2}$.

Another question is whether we really know the position of the neutron drip line. Let us first look at the theoretical and empirical arguments. Not so long ago it would have generally believed that mass formulas or extrapolations, what almost amounts to the same thing, could easily give the answer. We are more sceptical now and that for two reasons. The first is that we know that a dramatic change in structure takes place at the drip line so that the stability that we are seeking to predict will depend on features that are not part of the input. And the second is that neutron matter presumably is close to beeing bound, so that a neutron cluster coupled to a stable nuclear core will have a total energy close to zero " and with some luck negative. The systematics of nuclear one- and two neutron separation energies show evidence of such an effect for some elements: The curves seem to flatten out and to approach the x-axis almost tangentially [77]. It is clear that the demonstrations of stability obtained with the recoil separators are of capital importance for this knowledge.

Thus we are back to experiment as the only sure way to determine the position of the drip line. It is commonly believed that the neutron drip line has been mapped [78] up to neon $(Z=10)$, which very likely is true. It must be kept in mind, however, that the halo nuclei are very delicate objects that must be produced either in their ground state by a kind of Mössbauer effect ${ }^{\mathrm{n}}$, or not be produced at all. This

\footnotetext{
"A measure of the formation probability is the square of the overlap between a neutron pair in a normal nucleus (or reaction complex) and the pair of neutrons in the halo. Fortunately, this does not scale as $R^{-6}$, where $R$ is the halo radius, as has been suggested by Gibbs and Hayes [72], which would have meant that the crosssections would depend on the neutron separation energy $S$ as $S^{3}$. The correct relationship, found by Riisager et al. [34], is proportionality to $R^{-2}$ and hence to $S$.
} 
effect is not necessarily taken into account by a systematics of cross-sections as is seen from the example of ${ }^{14} \mathrm{Be}$ that was "proven" [79] non-existent in 1970. It is also thinkable [80] that there will be islands, or maybe better reefs, of stability in the sea of neutron instability, so that, to take a random example, ${ }^{25,26,27} \mathrm{O}$ would be unbound (as seems to be the case) and that, nevertheless, ${ }^{28} \mathrm{O}$ would be bound...

In conclusion let me say that I began by mentioning how theory with ease takes us from the known ${ }^{116} \mathrm{Sn}$ to the (experimentally inaccessible) ${ }^{176} \mathrm{Sn}$. In the real world it has taken five years and around 100 experimental and theoretical papers to begin bridging the gap between ${ }^{9} \mathrm{Li}$ and ${ }^{11} \mathrm{Li}$. We have all reasons to expect that further work in this region will be highly rewarding.

Acknowledgements. I am indebted to a number of colleagues and especially to Drs. A. Galonsky, K. Ieki, N.A. Orr and 1. Tanihata for information about recent experiments on the neutron halo.

\section{REWTRENCES}

1 K.Ikeda and Y. Suzuki (eds.), Structure and Reactions of Unstable Nuclei,World Scientific, Singapore 1991, pp 1-356.

2 C. Détraz and D.J. Vieira, Ann. Rev. Nucl. Part. Sci. 39 (1989) 407-465.

3 6th Int.Conf. on Nuclei Far From Stability, Bernkastel-Kues, Germany, 19-24 July 1992, Proceedings to be published.

4 R.D. Page, P.J. Woods, R.A. Cunningham, T. Davinson, N.J. Davis, S. Hofmann, A.N. James, K. Livingston, P.J. Sellin and A.C. Shotter, Phys. Rev. Lett. 68 (1992) 1287; see also paper presented at this conference.

5 V. Borrel, J.C. Jacmart, F. Pougheon, R.Anne, D. Détraz, D. GuillemaudMueller, A.C. Mueller, D. Bazin, R. Del Moral, J.P. Dufour, F. Hubert, M.S. Pravikoff and E. Roeckl, Nucl. Phys. A531 (1991) 353-369.

6 T. Bjørnstad, M.J.G Borge, P. Dessagne, R.D. von Dincklage, G.T. Ewan, P.G. Hansen, A. Huck, B. Jonson, G. Klotz, A. Knipper, P.O. Larsson, G. Nyman, H.L. Ravn, C. Richard-Serre, K. Riisager, D. Schardt and G. Walter, Nucl. Phys. A443 (1.985) 283; M.J.G. Borge, P. Dessagne, G.T. Ewan, P.G. Hansen, A. Huck, B. Jonson, G. Klotz, A. Knipper, S. Mattsson, G. Nyman, C. Richard-Serre, K. Riisager and G. Walter, Phys. Scripta 36 (1987) 218; M.J.G. Borge, P.G. Hansen, B. Jonson, S. Matsson, G. Nyman, A. Richter and K. Riisager, Z. Phys. A 332 (1989) 413.

7 M.J.G. Borge, H. Cronberg, M. Cronquist, H. Gabelmann, P.G. Hansen, L. Johannsen, B. Jonson, S. Mattsson, G. Nyman, A. Richter, K. Riisager, O. Tengblad and M. Tomaselli, Nucl. Phys. A490 (1988) 287.

8 P.G. Hansen and B. Jonson, Europhys. Lett. 4 (1987) 409.

9 T. Kobayashi, Nucl.Phys.A538 (1992) 343c; C. Thibault, R. Klapisch, C. Rigaud, A.M. Poskanzer, R. Prieels, L. Lessard and W. Reisdorf, Phys. Rev. C12 (1975) 644; J.M. Wouters, R.H. Kraus, D.J. Vieira, G.W. Butler and K.E.G. Löbner, Z. Phys. A 331 (1988) 229 
10 E. Arnold, J. Bonn, R. Gegenwart, W. Neu, R. Neugart, E.-W. Otten, G. Ulm and $\mathrm{K}$. Wendt, Phys. Lett. 197B (1987) 311.

11 E. Arnold, J. Bonn, A. Klein, R. Neugart, M. Neuroth, E.-W. Otten, P. Lievens, H. Reich and W. Widdra, Phys. Lett. 281B (1992) 16.

12 B. Blank, J.-J. Gaimard, H. Geissel, K..H. Schmidt, H. Stelzer, K. Sümmerer, D. Bazin, R. Del Moral, e.P. Dufour, A. Fleury, F. Hubert, H. G. Clerc and M. Steiner, Z. Phys. A340 (1991) 41-50; Preprints GST-92-22 (March 1992) and -92 41. (June 1992), to be published.

13 I. Tawihata, H. Hamagaki, O. Hashimoto,S. Nagamiya, Y. Shida, N. Yoshikawa, O. Yamakawa, K. Sugimoto, T. Kobayashi, D. Greiner, N. Takahashi, and Y. Nojiri, Phys. Lett.160B (1985) 380; I. Tanihata, H. Hamagaki, O. Hashimoto, Y. Shida, N. Yoshikawa, K. Sugimoto, O. Yamakawa, T. Kobayashi and N. Takahashi, Phys. Rev. Lett. 55 (1985) 2676.

14 M. Fukuda, T. Ichihara, N. Inabe, T. Kubo, H. Kumagai, T.Nakagawa, I. Tanihata, Y. Yano, M. Adachi, K. Asahi, M. Kouguchi, M. Ishihara, H. Sagawa and S. Shimoura, Phys. Lett. B268 (1991) 339.

15 H. Sagawa, Phys.Lett. B286 (1992) 7.

16 T. Kobayashi, O. Yamakawa, K. Omata, K. Sugimoto, T. Shimoda, N. Takahashi and I. Tanihata, Phys. Rev. Lett. 60 (1988) 2599.

17 H. Sagawa and K. Yazaki, Phys. Lett. 244B (1990) 149.

18 T. Kobayashi, S. Shimoura, Y. Tanihata, K. Katori, K. Matsuta, T. Minamisono, K. Sugimoto, W. Müller, D.L. Olson, T.J.M. Symons, and H. Wieman, Phys. Lett $2328(1989) 51$.

19 R. Anne, S.E. Amell, R. Bimbot, H. Emling, D. Guillemaud-Mueller, P.G. Hansen, L. Johamnen, B. Jonson, M. Lewitowicz, S. Mattsson, A.C. Mueller, R. Neugart, G. Nyman, F. Pougheon, A. Richter, K. Riisager, M.G. Saint-Laurent, G. Schrieder, O. Sorlin and K. Wilhelmsen, Phys. Lett. $250 \mathrm{~B}$ (1990) 19-23.

20 I. Tanihata, D. Hirata, T. Kobayashi, S. Shimoura, K. Sugimoto and H. Toki, preprint RIKEN-AF-NP-123 (April 1992) and to be published.

21 K. Yabana, Y. Ogawa and Y. Suzuki, Nucl. Phys. A539 (1992) 295-315.

22 M.J.G. Borge, P.G. Hansen, L. Johannsen, B. Jonson, T. Nilsson, G. Nyman, A. Richter, K. Riisager, $O$. Tengblad, K. Wilhelmsen and the ISOLDE Collaboration, Z.Phys. A340 (1991) 255.

23 M.J.G. Borge, M. Epherre-Rey-Campagnolle, D. Guillemaud-Mueller, B. Jonson, M. Langevin, G. Nyman and C. Thibault, Nucl. Phys A460 (1986) 373; K. Riisager et al. in [3].

24. J.C. Slater, Quantum Theory of Matter, McGraw-Hill, N.Y. 1968, p 434.

25 B. Lohmann and E. Weingold, Phys. Lett. 86A (1981) 139.

26 A. Martin, J.-M. Richard and T.T. Wu, CERN-TH 6227/91 (1991) and to be published in J.Chem.Phys.; A. Martin CERN-TH 6376/92 (1992).

27 A.P. Mills, Phys. Rev. Lett. 46 (1981) 717.

28 L.D. Landau and E.M. Lifshitz, Quantum Mechanics (Non-Relativistic Theory), Pergamon Press, 3rd ed., Oxford, 1977, p. 114.

29 J.M. Levy-Leblond, Phys. Rev. 153 (1967) 1.

30 K.R. Lykke, R.D. Mead and W.C. Lineberger, Phys. Rev. Lett. 52 (1984) 2221.

31 V.M. Efimov, Comments Nucl. Part. Phys. 19 (1990) 271. 
32 J. Macek, J. Phys. D3 (1986) 31: Z. Zhen and J. Macek, Phys. Rev A38 (1988) 1193.

33 A.I. Amelin, M.G. Gornov, Yu.B. Gurov, A.L. Il'in, P.V. Morokhov, V.A. Pechkurov, V.I. Savel'ev, F.M. Sergeev, S.A. Smirnov, B.A. Chernyshev, R.R. Shafigulin and V.A. Shishkov, Sov. J. Nucl. Phys. 52 (1990) 782; H.G. Bohlen et al. in [3].

34 K. Riisager, A.S. Jensen and P. Møller, Two-body halos, Preprint February 1992 , to be published in Nuclear Physics.

35 I. Talmi and I. Unna, Phys. Rev. Lett. 4 (1960) 469.

36 T. Otsuka, N. Fukunishi and H. Sagawa, Ref. [1] p. 100.

37 D.J. Millener, J.W. Olness, E.K. Warburton and S.S. Hanna, Phys. Rev. C 28 (1983) 497.

38 M.V. Zhukov, B.V. Danilin, A.A. Korshennikov and L.V. Chulkov, Europhys. Lett. 12 (1990) 307.

39 G.R. Satchler, K.W. McVoy and M.S. Husssein, Nucl. Phys.A522 (1991) 621.

40 B.-A. Li, M.S. Hussein and W. Bauer, Nucl. Phys. A533 (1991) 749.

41 N. Takigawa and H. Sagawa, Phys. Lett B265 (1991) 23.

42 M.V. Zhukov,L.V. Chulkov, D.V. Fedorov, B.V. Danilin, J.M. Bang, J.S. Vaagen and I.J. Thompson, NORDITA preprint $92 / 29 \mathrm{~N}$ and to be published.

43 Y. Suzuki and Y. Tosaka, Nucl. Phys. 517 (1990) 599-614.

44 C.A. Bertulani and G. Baur, Nucl. Phys. A480 (1988) 615-628.

45 A.N.F. Alexio and C.A. Bertulani, Nucl. Phys. A505 (1989) 448.

46 M. Honma and H. Sagawa, Progr. Theor. Phys. 84 (1990) 494.

47 G. Bertsch and J. Foxwell, Phys. Rev. C41 (1990) 1300 and C42 (1990) 1159.

48 C.A. Bertulani and G. Baur, Nucl. Phys. A526 (1991) 751-761.

49 G. Baur, in ref. [1] p.211.

50 C.A. Bertulani and L.F. Canto, Nucl. Phys. A539 (1992) 163.

51 K. Suotome, S. Yamaji and M. Sano, Nucl. Phys. A538 (1992) 383c.

52 H. Esbensen, Phys. Rev. C44 (1991) 440.

53 H. Esbensen and G.F. Bertsch, Soft Dipole Excitations in ${ }^{11}$ L, Argonne Preprint PHY-6888-TH-91, and to be published in Nucl.Phys.

54 R. Anne, S.E. Arnell, R. Bimbot, S. Dogny, H. Emling, D. Guillemaud-Mueller, P.G. Hansen, P. Hornshøj, F. Humbert, B. Jonson, M. Lewitowicz, A.C. Mueller, P. Møller, R. Neugart, G. Nyman, T. Nilsson, F. Pougheon, A. Richard, A. Richter, K. Riisager, M.G. Saint-Laurent, G. Schrieder, O. Sorlin and K. Wilhelmsen, Experiment on ${ }^{11} \mathrm{Li}$ and ${ }^{11} \mathrm{Be}$, to be published (1992).

55 R.J. Glauber, Phys.Rev. 99 (1955) 1515.

56 T. Kobayashi in Proc. of the First Radioactive Nuclear Beams Conference, Eds. W.D. Myers et al. (World Scientific, Singapore, 1990).

57 B. Alex Brown, NP A522 (1991) 221c-239c.

58 G.F. Bertsch, B.A. Brown and H. Sagawa, Phys. Rev. C39 (1989) 1154.

59 G. Bertsch, H. Esbensen and A. Sustich, Phys. Rev. C42 (1990) 758.

60 L.Johannsen, A.S. Jensen and P.G. Hansen, Phys. Lett. 244B (1990) 357; L. Johannssen, The Halo of ${ }^{11} \mathrm{Li}, \mathrm{Ph}$. D. Thesis Institute of Physics and Astronomy. Aarhus University (1991), pp. 1-80.

61 Y. Tosaka, Y. Suzuki and K. Ikeda, Progr. Theor. Phys. 83 (1990) 1140. 
62 Z. Ren and G. Xu, Phys. Lett. B252 (1990) 311.

63 G.F. Bertsch and H. Esbensen, Ann. Phys. 209 (1991) 327

64 M. Lassaut and R.e. Lombard, Z.Phys. A341 (1992) 125.

65 M.V. Zhukov, B.V. Danilin, D.V. Fedorov, J.S. Vaagen, F.A. Gareev and J.M. Bang, Phys. Lett. B265 (1991) 19.

66 M.V. Zhukov, D.V. Fedorov, B.V. Danilin, J.S. Vaagen and J.M. Bang, Nucl. Phys. A539 (1992) 295.

67 H. Sagawa, N. Takigawa and Nguyen van Giai, Nucl. Phys. A543 (1992) 575.

68 I. Tanihata, ref. [1] p. 233.

69 I. Tanihata, T. Kobayashi, T. Suzuki, K. Yoshida, S. Shimoura, K. Sugimoto, K. Matsuta, T. Minamisono, W. Christie, D. Olson, and H. Wieman, Determination of the density distributions and the correlation of halo neutrons ${ }^{i n}{ }^{11} \mathrm{Li}$, RIKEN. AF-NP-119, January 1992 and to be published.

70 K. Riisager, R. Anne, S.E. Arnell, R. Bimbot, H. Emling, D. GuillemaudMueller, P.G. Hansen, L. Johannsen, B. Jonson, A. Latimier, M. Lewitowicz, S. Mattsson, A.C. Mueller, R. Neugart, G. Nyman, F. Pougheon, A. Richard, A. Richter, M.G. Saint-Laurent, G. Schrieder, O. Sorlin and K. Wilhelmsen, Nuclear Physics A540 (1992) 365.

71 N.A. Orr, N. Anantaraman, Sarn M. Austin, C.A. Bertulani, K. Hanold, J.H. Kelley, D.J. Morrisey, B.M. Sherrill, G.A. Souliotis, M. Thoennessen, J.S. Winfield and J.A. Winger, Momentum distributions of ${ }^{9} \mathrm{Li}$ fragments following the breakup of ${ }^{11} \mathrm{Li}$, to be published.

72 S. Shimoura, T. Nakamura, M. Ishihara, N. Inabe, T. Kobayshi, T. Kubo, R.H. Siemssen, I. Tanihata and Y. Watanabe, E1 Strength Distribution of ${ }^{11} \mathrm{Li}$ by Invariant Mass Spectroscopy, in [3].

73 C.B. Moon, M. Fujimaki, N. Inabe, K. Katori, T. Kobayashi, H. Kumagai, T. Kubo, S. Shimoura, T. Suzuki and I. Tanihata, ${ }^{9,11} \mathrm{Li}+\mathrm{p}$ Elastic Scattering, in [3].

$74 \mathrm{M}$. Lewitowicz et al., Elastic Scattering of $29 \mathrm{MeV} / \mathrm{n}^{11} \mathrm{Li}$ on a Si Target, in [3].

75 W.R. Gibbs and A.C. Hayes, Phys.Rev.Lett. 67 (1991) 1395.

76 Y. Suzuki and K. Yabana, Phys. Lett. B272 (1991. 173

77 G. Audi, Nuclear Mass Update 1992, personal communication.

78 D. Guillemaud-Mueller, J.C. Jacmart, E. Kashy, A. Latimier, A.C. Mueller, F. Pougheon, A. Richard, Yu.E. Penionzhkevich, A.G. Artukh, A.V. Belozyorov, S.M. Lukyanov, R. Anne, P. Bricault, C. Détraz, M. Lewitowics, Y. Zhang, Yu.S. Lyutostansky, M.V. Zverev, D. Bazin and W.D. Schmitt-Ott, Phys.Rev.C 41 (1990) 937.

79 A.G. Artukh et al., Phys.Lett. 33B (1970) 407.

80 A.S. Jensen and K. Riisager, Stable nuclear Structure Along the Neutron Drip Line?, Nucl.Phys.A (in press). 
